\title{
Técnica e resultados da endarterectomia de artéria coronária
}

\author{
Pedro R. SALERNO*, Jarbas J. DINKHUYSEN*, Paulo CHACCUR*, Camilo ABDULMASSIH NETO*, \\ Magaly A. SANTOS*, Luiz Carlos Bento de SOUZA*, Adib D. JATENE*
}

RBCCV 44205-244

SALERNO,P.R.; DINKHUYSEN, J. J.; CHACCUR,P.; ABDULMASSIHNETO, C.; SANTOS, M. A.; SOUZA, L. C. B.; JATENE, A. D. - Técnica e resultados da endarterectomia de artéria coronária. Rev. Bras. Cir. Cardiovasc., 9 (3): 152-158, 1994.

RESUMO: A abordagem cirúrgica da doença coronária sofreu grandes modificaçōes nos últimos anos. Devido à possibilidade de angioplastia, os doentes encaminhados para cirúrgia sāo aqueles com doença ateromatosa difusa grave e/ou com artérias ocluídas, geralmente responsável por uma área de músculo viável. Assim sendo, a endarterectomia de coronária é um recurso técnico que viabiliza a abordagem destes vasos. Em nosso Serviço, no Hospital do Coração, foram submetidos para cirúrgia de revascularização do miocárdio (RM) com endarterectomia, no período de janeiro de 88 a dezembro de 92,110 pacientes(pts.). 0 sexo masculino predominou, com $99(90 \%)$ pts. Encontramos com função ventricular normal $33(30 \%)$, déficit moderado $71(64,5 \%)$ e severo $6(5,4 \%)$. Doze $(10,9 \%)$ pts. eram reintervenção para nova RM. Dividimos em 2 grupos quanto ao número de endarterectomias realizadas.Grupo A com uma endarterectomia $104(94,5 \%)$ pts. e Grupo B com mais de uma endarterectomia $6(5,4 \%)$ pts. No Grupo A a coronária esquerda (CE) foi abordada em $38(36,5 \%)$ pts. e a coronária direita (CD) $66(63,4 \%)$ pts. No Grupo B com mais de uma endarterectomia a CE foi abordada 8 vezes e a CD outras 4 . Em todos os casos o cirurgiāo removeu a endoartéria com a placa ateromatosa, com sucesso. A ocorrência de infarto trans-operatório na região da artéria endarterectomizada foi de $7(6,3 \%)$ pts. e em regiōes não relacionadas com as artérias manipuladas foi de $3(2,7)$ pts. Em $9(8,1 \%)$ pts. foi realizado procedimento associado, como: aneurismectomia do ventrículo esquerdo $5(4,5 \%)$ pts., troca de valva aórtica $1(0,9 \%)$ e ventrículotomia para retirada de trombo em $3(2,7 \%)$. As complicações mais freqūentes foram: arritmias $26(23,6 \%)$ pts., insuficiência renal aguda $10(9 \%)$ pts., síndrome de baixo débito (SBD) $4(3,6 \%)$ pts. Ocorreram $5(4,5 \%)$ óbitos, tendo como causa mais freqũente a SBD. Quatro $(3,8 \%)$ pts., do Grupo A e $1(16,6 \%)$ do Grupo B. Com esses resultados, verificamos que a endarterectomia é um procedimento que, utilizado criteriosamente, possibilita uma RM completa e com resultados consistentes.

DESCRITORES: artéria coronária, revascularização, cirurgia.

\section{INTRODUÇÃO}

Há pelo menos 50 anos, técnicas de revascularização do miocárdio (RM) vêm sendo desenvolvidas.

Em 1929, BECK 1,2 procurou aumentar o fluxo sangũíneo miocárdico, utilizando irrigação do pericárdio com substância irritante. Em 1951, VINEBERG ${ }^{25}$ e VINEBERG \& MILLER ${ }^{26}$ descre- veram o implante da artéria torácica interna no miocárdio. Neste mesmo período, outras tentativas também se destacaram, como omentopexia, a endarterectomia e arteriotomia com ampliação do vaso.

LONGMIRE Jr. et alii ${ }^{19}$ descreveram, em 1958, 5 casos de endarterectomia sob visão direta, em pacientes anginosos, sem infarto prévio, obtendo melhora clínica em 4. Em 1959, surgiu a cineco-

Trabalho realizado no Hospital do Coração da Associação do Sanatório Sírio. São Paulo, SP. Brasil.

Apresentado ao $21^{\circ}$ Congresso Nacional de Cirurgia Cardiaca. Porto Alegre, RS, 24 a 26 de março, 1994.

* Do Hospital do Coração da Associação do Sanatório Sírio.

Endereço para correspondência: Pedro Salerno. Rua Desembargador Eliseu Guilherme, 123. Paraiso. 04004-030 São Paulo, SP, Brasil. 
SALERNO, P. R.; DINKHUYSEN, J. J.; CHACCUR, P.; ABDULMASSIH NETO, C.; SANTOS, M. A.; SOUZA, L. C. B.; JATENE, A. D. - Técnica e resultados da endarterectomia de artéria coronária: Rev. Bras. Cir. Cardiovasc., 9 (3): 152-158, 1994.

ronariografia, desenvolvida por SONES Jr. ${ }^{24}$, tornando possivel a identificação in vivo das lesões ateroscleróticas coronarianas. Tal tecnologia lançou as bases para a cirurgia atual das artérias coronárias.

Por quase 3 décadas, a RM tem sido utilizada com sucesso. Na maioria dos pacientes, o efeito hemodinâmico de lesões coronárias proximais pode ser eliminado através do enxerto venoso ou arterial. Entretanto, em $10 \%$ a $20 \%$ dos pacientes, o processo aterosclerótico envolve o leito distal, obstruindo vasos tributários, de maneira que a efetividade do enxerto fica comprometida ${ }^{18}$. Com o uso em larga escala da angioplastia com balão para as lesões proximais, e a necessidade cada vez maior de reoperações, prevê-se um número crescente de indivíduos com lesões coronárias difusas e leito distal ruim. Por outro lado, a exploração intra-operatória das artérias coronárias pode revelar um comprometimento difuso, maior que o sugerido pela angiografia. Nestes casos, a endarterectomia surge como um procedimento coadjuvante dentro das técnicas de revascularização do miocárdio. Os resultados deste procedimento têm sido questionados por aumentar o risco cirúrgico 5,23 . Controvérsias existem em relação às indicações, seleção dos pacientes e os benefícios do mesmo.

Neste estudo, procuramos delinear os aspectos de indicação, técnica e resultados imediatos.

\section{CASUÍSTICA E MÉTODOS}

No período entre janeiro de 1988 e dezembro de 1992 , foram realizadas 2781 operações de revascularização do miocárdio (RM) no Hospital do Coração - Associação do Sanatório Sírio.

Deste total, $110(3,9 \%)$ pacientes foram submetidos a RM associado a endarterectomia de coronária. A idade, neste grupo de pacientes, foi de 35 a 80 anos, com idade média de 58,9 anos. Noventa e nove $(90 \%)$ pacientes eram do sexo masculino e $11(10 \%)$ do sexo feminino.

Dividimos em 2 grupos, quanto ao número de endarterectomias realizadas. Grupo A com 1 endarterectomia $104(94,5 \%)$ pacientes. Grupo B com mais de 1 endarterectomia $6(5,5 \%)$ pacientes.

No Grupo A, com 104 pacientes, a coronária direita foi abordada em 66 pacientes e a coronária esquerda em 38 pacientes. No Grupo B com 6 pacientes, tivemos 2 pacientes com endarterectomia de coronária direita e 4 pacientes de coronária esquerda (Tabela 1).

Considerando como fatores de risco cirúrgico a disfunção ventricular, reoperação, diabetes mellitus insulino-dependente, sexo feminino e idade superior
TABELA 1

GRUPO E LOCAL ENDARTERECTOMIA

\begin{tabular}{cccccc}
\hline & \multicolumn{2}{c}{$C D$} & & \multicolumn{2}{c}{ CE } \\
\cline { 2 - 3 } \cline { 5 - 6 } & $N^{\circ}$ & TOTAL $\%$ & & $N^{\circ}$ & TOTAL \% \\
Grupo A & $66 / 104$ & 63,4 & & $38 / 104$ & 36,5 \\
Grupo B & $2 / 6$ & 33,3 & & $4 / 6$ & 66,6 \\
\hline
\end{tabular}

a 70 anos, dividimos os pacientes em 3 grupos. 0 Grupo 1 composto por 77 pacientes que não possuiam nenhum fator de risco; Grupo 2 com 27 pacientes e um fator de risco presente e o Grupo 3 com 6 pacientes com mais de um fator de risco cirúrgico (Tabela 2).

A função ventricular foi considerada normal (F.E. $>0,50)$ em $33(30 \%)$ dos pacientes, com comprometimento moderado (F.E. $0,30-0,50)$ em $71(64,5 \%)$ pacientes e severo (F.E. $<0,30)$ em $6(5,4 \%)$ pacientes. Lesões do tronco da coronária esquerda estiveram presentes em $9(8,1 \%)$ pacientes. Doze $(10,9 \%)$ pacientes tinham cirúrgia de revascularização do miocárdio prévia.

Durante a mesma intervenção foram realizados procedimentos associados, tais como: aneurismectomia de ventrículo esquerdo em $5(4,5 \%)$ pacientes, $1(0,9 \%)$ paciente com substituição da valva aórtica, e trombectomia do ventrículo esquerdo em $3(2,7 \%)$ pacientes.

A técnica empregada em todos os pacientes foi a circulação extracorpórea com hipotermia moderada e pinçamento aórtico intermitente.

A média de pontes revascularizadas foi de 3,5/ pacientes, sendo que das 384 pontes $30 \%$ foram realizadas em leitos endarterectomizados.

Utilizamos a técnica de extração manual do cordão ateromatoso para a realização da endarterectomia. Após a arteriotomia isola-se o cordão ateromatoso, realizando-se sua extração com uma tração suave, mantida e praticada pelo cirurgião, e uma contração na superfície epimiocárdica, evitando pinçar os ramos arteriais, manobra realizada pelo assistente. O detalhe técnico é semelhante para

TABELA 2

FATORES DE RISCO

\begin{tabular}{ccc}
\hline FATORES DE RISCO & PACIENTES & $\%$ \\
\hline Grupo 1 & 77 & 70 \\
Grupo 2 & 27 & 24,5 \\
Grupo 3 & 6 & 5,4 \\
\hline Total & 110 & $100 \%$ \\
\hline
\end{tabular}


SALERNO, P. R.; DINKHUYSEN, J. J.; CHACCUR, P.; ABDULMASSIH NETO, C.; SANTOS, M. A.; SOUZA, L. C. B.; JATENE, A.

D. - Técnica e resultados da endarterectomia de artéria coronária: Rev. Bras. Cir. Cardiovasc., 9 (3): 152-158, 1994.

porção proximal, porém sem a preocupação de retirar todo o cordão ateromatoso. $\mathrm{Na}$ eventualidade de fratura do cordão ateromatoso, praticamos uma arteriotomia mais distal, a fim de retomarmos a extração do cordão ateromatoso e evitarmos a oclusão arterial. O fluxo distal é sempre verificado através da injeção de solução salina com seringa pelo enxerto, ou pela fluxometria aferida pela pressão pré-cânula ${ }^{12}$. A revascularização do leito endarterectomizado é feita de maneira convencional, com enxerto venoso ou arterial.

\section{RESULTADOS}

A mortalidade hospitalar ocorreu em 5 (4,5\%) pacientes, 4 por insuficiência miocárdica e 1 por falência de multiplos órgãos. Embora a mortalidade fosse mais acentuada nos grupos com mais fatores de risco, não houve significância estatística para este evento, provavelmente devido ao pequeno número de pacientes do Grupo 3 (Tabela 3 ).

TABELA 3

FATOR DE RISCO X ÓBITO

\begin{tabular}{cccc}
\hline FATORES DE RISCO & PACIENTES & OBITO & $\%$ \\
\hline Grupo 1 & 77 & 2 & 2,5 \\
Grupo 2 & 27 & 1 & 3,7 \\
Grupo 3 & 6 & 2 & 33,3 \\
\hline Total & 110 & 5 & $4,5 \%$ \\
\hline
\end{tabular}

- Não significativo

$(P<0.05)$

A correlação entre os fatores de risco cirúrgico com os Grupos A e B, uma e mais de uma endarterectomia, respectivamente, revelou que a mortalidade total no Grupo A foi de $3,8 \%$, enquanto que no Grupo B foi de $16,6 \%$, ० que não é, estatísticamente, significativo (Tabela 4).
A análise do Grupo A, com 106 pacientes, e o local da endarterectomia mostraram que 4 pacientes com endarterectomia da coronária direita foram a óbito, enquanto nenhum óbito ocorreu quando a coronária esquerda sofreu endarterectomia (Tabela 5).

Infarto per-operatório, diagnosticado pela presença de nova onda " $q$ " no eletrocardiograma e pela curva da enzima CKMB, ocorreu em 10 pacientes $(9,0 \%)$; destes, em $7(6,3 \%)$ pacientes a área infartada estava relacionada com o local da endarterectomia e em $3(2,7 \%)$ ocorreu em outra área.

Nos 7 pacientes, nos quais o infarto agudo do miocárdio ocorreu na área endarterectomizada, a cinecoronariografia pré-operatória mostrava que a artéria já estava ocluída em 3 deles.

As complicações mais encontradas foram: arritmias em $26(23,6 \%)$ pacientes, drenagem sangüínea aumentada em $12(10,9 \%)$, insuficiência renal aguda em $10(9,0 \%)$, síndrome de baixo débito em $4(3,6 \%)$. Como terapêutica coadjuvante, utilizamos a ultrafiltração em 1 paciente e balão intra-órtico em outro.

\section{COMENTÁRIOS}

A revascularização do miocárdio (RM) na doença aterosclerótica coronária cursa com bons resultados. Porém, em cerca de $10 \%-20 \%$ dos pacientes, o processo aterosclerótico envolve o leito distal, obstruindo vasos tributários, de maneira que a efetividade do enxerto fica comprometida ${ }^{18}$. A endarterectomia pode ser empregada nestes casos, pois permite uma revascularização mais completa para áreas com músculo viável. $O$ valor da revascularização completa é bem documentado. Os pacientes evoluem com desaparecimento dos sintomas,menor incidência de reoperaçōes e uma taxa de sobrevida maior quando comparados com

TABELA 4

FATORES DE RISCO X GRUPOS

\begin{tabular}{|c|c|c|c|c|c|c|}
\hline \multirow[b]{2}{*}{ FATORES DE RISCO } & \multicolumn{3}{|c|}{ GRUPO A } & \multicolumn{3}{|c|}{ GRUPO B } \\
\hline & ОВІтО & TOTAL & $\%$ & ОВВІто & TOTAL & $\%$ \\
\hline $\begin{array}{l}\text { Grupo } 1 \\
\text { Grupo } 2 \\
\text { Grupo } 3\end{array}$ & $\begin{array}{l}2 \\
0 \\
2\end{array}$ & $\begin{array}{c}73 \\
25 \\
6\end{array}$ & $\begin{array}{c}2,5 \\
- \\
33,3\end{array}$ & $\begin{array}{l}0 \\
1 \\
-\end{array}$ & $\begin{array}{l}4 \\
2 \\
-\end{array}$ & 50 \\
\hline Total & 4 & 104 & 3,8 & 1 & 6 & 16,6 \\
\hline
\end{tabular}

* não significativo

$(\mathrm{P}<0.05)$ 
SALERNO, P. R.; DINKHUYSEN, J.J.; CHACCUR, P.; ABDULMASSIH NETO, C.; SANTOS, M. A.; SOUZA, L. C. B.; JATENE, A. D. - Técnica e resultados da endarterectomia de artéria coronária: Rev. Bras. Cir. Cardiovasc., 9 (3): 152-158, 1994.

TABELA 5

GRUPO A X LOCAL ENDARTERECTOMIA

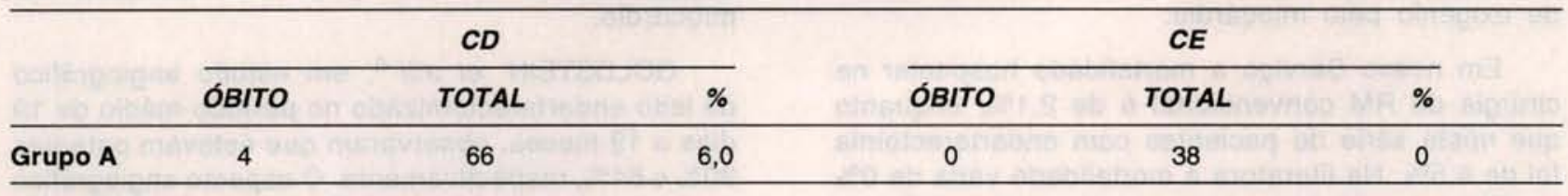

- não significativo

$(P<0,05)$

a revascularização incompleta $(8,9 \%)$. LAWRIE et alii 16 estudaram 1274 homens com doença aterosclerótica em 2 ou 3 coronárias, que se submeteram à RM. Dentre os resultados obtidos, o fator preditivo que mais influênciou na mortalidade tardia foi o número de artérias não tratadas 16. SCHAFF et alii ${ }^{23}$ estudaram o resultado de 10 a 12 anos de RM em 500 pacientes e verificaram que a presença de artérias com doença e que não receberam pontes foi um dos importantes fatores preditivos de eventos cardíacos tardios, incluindo óbito de causa cardíaca, recorrência de angina, infarto do miocárdio e reoperações.

A primeira tentativa de revascularização do miocárdio começou com a endarterectomia feita com curetagem intraluminal ou sob visão direta das placas localizadas 2, 19 . Estes procedimentos iniciais foram realizados sem os benefícios da circulação extracorpórea e da arteriografia coronária. Apesar da utilização destes recursos no início dos anos 60 , a endarterectomia foi empregada com pouca freqüência e com resultados inconsistentes 6,7 . Estes resultados melhoraram quando a endarterectomia foi associada a revascularização do leito endarterectomizado 9,15 .

Para realizar a endarterectomia, pode-se utilizar métodos manuais ou o gás monóxido de carbono. SAWYER et alii ${ }^{15}$ descrevem o uso do gás, porém outros trabalhos demonstraram não haver benefícios desta técnica em relação à manual $9,11,15$. Desde 1972, a endarterectomia de coronária tem sido empregada por muitos cirurgiões na coronária direita $(C D)$ e, mais seletivamente, para o sistema da coronária esquerda (CE) 17,18 .

A seleção dos pacientes baseia-se nas evidências clínicas de isquemia miocárdica e pela confirmação angiográfica do envolvimento importante do leito distal coronário. Nesta situação a indicação é considerada eletiva, mas em alguns casos será circunstancial, ou seja, o cirurgião encontrará um leito ateromatoso difuso com ramos não passíveis de receber pontes, mas que suprem uma grande área de miocárdio isquêmico. A indicação mais freqüente para a endarterectomia é para os casos de coronária direita $(C D)$ dominante ocluída responsá- vel por uma área de miocárdio viável. A decisão para o sistema da coronária esquerda (CE) deve ser bem criteriosa, devido às dificuldades técnicas citadas por vários autores $13,20,21$. As contra-indicações para a utilização de endarterectomia são vasos com pequeno calibre $(<1.0 \mathrm{~mm})$, vasos de áreas com fibrose devido a infarto prévio, artérias coronárias aneurismáticas e artérias intensamente calcificadas 17,20 .

Segundo BRENOWITZ et alii ${ }^{3}$, a disfunção ventrícular severa, reoperação, diabetes mellitus insulino-dependente, sexo feminino e idade superior a 70 anos, adicionam mais riscos quanto mais fatores estiverem presentes, principalmente quando mais de uma endarterectomia é realizada.

As características anatômicas da coronária direita como a dominância, o maior calibre, acesso ao tronco e tributária mais fácil, fazem com que os resultados sejam mais favoráveis do que na coronária esquerda $3,9,18,21$. Em nossa casuística, a mortalidade foi maior nos pacientes em endarterectomia da CD $4(6 \%)$ pacientes do que na $C E$, onde não tivemos óbitos ( $0 / 41$ pacientes). Porém, este valor não é estatisticamente significativo. Atribuímos este resultado ao fato de que, dentre os 4 pacientes que faleceram, 2 possuiam mais de um fator de risco, - que colabora para aumentar a mortalidade ${ }^{3}$; em outro o padrão arterial era ruim e evoluiu com infarto transoperatório e oclusão da artéria, e no último a causa não foi cardíaca. Além disso, acreditamos que a seleção dos pacientes para a realização da endarterectomia da CE foi mais criteriosa, baseando-se no fato anatômico da artéria não ser favorável para o procedimento 9 .

$\mathrm{O}$ infarto per-operatório ocorre com mais freqüência nos pacientes com endarterectomia do que nos pacientes com RM convencional $3,4,9,18$, o que se confirmou em nossa série $(2,8 \%$ na cirurgia convencional, 9,0\% com endarterectomia de coronária). Em $6,3 \%$ a área infartada estava relacionada com a artéria endarterectomizada.

QUERESHI et alii ${ }^{21}$ mostraram que, nos casos onde ocorreu infarto do miocárdio transoperatório, a oclusão da ponte ocorreu em $39 \%$ dos casos, o que significa que, na maioria deles, foi por oclusão 
SALERNO, P. R.; DINKHUYSEN, J. J.; CHACCUR, P.; ABDULMASSIH NETO, C.; SANTOS, M. A.; SOUZA, L. C. B.; JATENE, A. D. - Técnica e resultados da endarterectomia de artéria coronária: Rev. Bras. Cir. Cardiovasc., 9 (3): 152-158, 1994.

de ramos intra-miocárdicos, embolização, ou por um período de desequilíbrio entre oferta e consumo de oxigênio pelo miocárdio.

Em nosso Serviço a mortalidade hospitalar na cirurgia de RM convencional é de $2,1 \%$, enquanto que nesta série de pacientes com endarterectomia foi de $4,5 \%$. Na literatura a mortalidade varia de $0 \%$ a $30 \%$ quando os pacientes são submetidos à endarterectomia $11,13,14$, pois têm um padrão arterial ruim e na evolução a ocorrência de eventos cardíacos é elevada 16,23 .

Quanto ao enxerto patente no leito endarterectomizado, BRENOWITZ et alii ${ }^{3}$ mostram que em 1 ano, $71,1 \%$ das pontes com endarterectomia estão pérvias e $75,8 \%$ para as pontes convencionais; na evolução de 58,3 meses a recorrência de angina ocorreu em $28,9 \%$ dos pacientes com cirurgia convencional, $32,5 \%$ para uma endarterectomia e $33,7 \%$ com mais de uma endarterectomia, o que não é estatísticamen-te significativo.

QURESHI et alii ${ }^{21}$ analizaram of fluxo nas pontes realizadas no sistema da coronária esquerda, com endarterectomia no $1^{2}$ ou mais anos após a operação e verificaram que em $76 \%$ era bom, $14 \%$ moderado e $10 \%$ ruim. Isto mostra que na grande maioria dos pacientes é possível não reabilitar o leito coronário, mas permitir um fluxo adequado ao miocárdio.

GOLDSTEIN et alii ${ }^{8}$, em estudo angiográfico do leito endarterectomizado no período médio de 19 dias a 19 meses, observaram que estavam patentes $90 \%$ e $64 \%$, respectivamente. O aspecto angiográfico tardio é de um vaso que sofre um processo sugestivo de fibrose.

WALLEY et alii ${ }^{27}$, em um estudo das alterações seqüênciais na morfologia das artérias endarterectomizadas, mostraram que, no período que variou desde o pós-operatório imediato até acima de cinco anos, ocorre um processo de proliferação miocintimal eoncêntrica e progressiva. A estenose severa nos vasos endarterectomizados é incomum e é vista apenas nos pacientes com mais de cinco anos após a operação. Estes resultados correspondem a aspectos encontrados em outras séries 24, 14 .

Acreditamos que a endarterectomia, apesar de acrescentar maior morbi-mortalidade, é uma técnica útil a ser empregada na revascularização do miocárdio de pacientes que não seriam candidatos por possuírem doença aterosclerótica difusa e severa.

SALERNO, P.R.; DINKHUYSEN, J.J.; CHACCUR, P.; ABDULMASSIHNETO, C.; SANTOS, M. A.; SOUZA L. C. B.; JATENE, A. D. - Technique and results of coronary endarterectomy. Rev. Bras. Cir. Cardiovasc., 9 (3): 152-158, 1994.

ABSTRACT: The surgical approach to coronary artery disease has improved a lot during the past two decades. With the widespread use of balloon angioplasty, a growing percentage of surgical patients, have complex coronary pathology. In properly selected patients, coronary endarterectomy has been a valuable adjunt to bypass techiniques by removing obstructing lesions and preparing the distal artery for bypass grafting. Over a 5 years period (1988 to 1993), 2781 patients underwent surgical revascularization at our Instituition (Hospital do Coraçāo). Coronary artery bypass combined with coronary endarterectomy was done in 110 patients. There were 99 men $(90 \%)$ and 11 women, mean age 58.9 years. For comparation patients were placed into two groups: Group A, patients undergoing endarterectomy to one artery, 104 patients - $94.5 \%$. Group B, patients with more than one endarterectomy - 6 patients $5.4 \%$. Perioperative myocardial infarction envolving the area supplied by the endarterectomized artery occurred in $6.3 \%$, (7 patients). In $3(2.7 \%)$ the infarction was not related with the endarterectomized artery. Complications during the post-operative period were: arrhythmia in 26 patients $(23.6 \%)$, return to the operation room for control of bleeding in $12(10.9 \%)$, acute renal failure in $10(9 \%)$, and low cardiac output in $4(3.6 \%)$ patients. Operative mortality rate was $4.5 \%(5 / 110)$, due to low cardiac output and multiple organs failure; 4 (3.8\%) patients belonged to Group $A$ and 1 to Group B (16.6\%). This study demostrates that multiple bypass grafting and adjunt coronary endarterectomy, can yeld good clinical results in patients with difuse coronary artery disease, many of whom would otherwise be inoperable.

DESCRIPTORS: coronary artery, revascularization, surgery.

\footnotetext{
* Os testes estatísticos empregados foram Qui-Quadrado e o teste exato de Fisher.
} 
SALERNO, P. R.; DINKHUYSEN, J. J.; CHACCUR, P.; ABDULMASSIH NETO, C.; SANTOS, M. A.; SOUZA, L. C. B.; JATENE, A. D. - Técnica e resultados da endarterectomia de artéria coronária: Rev. Bras. Cir. Cardiovasc., 9 (3): $152-158,1994$.

AGRADECIMENTOS: Agradecemos ao Dr. Nagib Haddad pelas análises estatísticas realizadas, ao Dr. Leopoldo Piegas pelo fornecimento dos dados clínicos e à Srta. Gislene Carvalho Silles pelo empenho na realização deste manuscrito.

\section{REFERÊNCIAS BIBLIOGRÁFICAS}

1 BECK, C. S. - The effect of surgical solution of chlorimated soda (Dakin Solution) in the pericardial cavity. Arch. Surg., 18: 1959, 1929.

2 BECK, C. S. - Revascularization of the heart by grafting a systemic artery or a new branch from the aorta into the coronary sinus. JAMA, 137: $436,1948$.

3 BRENOWITZ, J. B.; KAYSER, K. L.; JOHNSON, W. D. - Results of coronary artery endarterectomy and reconstruction. J. Thorac. Cardiovasc. Surg., 95: 1-10, 1988.

4 CHENVECHAI, C.; GROVES, L. K.; REYES, E. A.; SHIREY, E. K.; SONES, M. F. - Manual coronary endarterectomy: clinical experience in $\mathbf{3 1 5}$ patients. J. Thorac. Cardiovasc. Surg., 70: 524-531, 1975.

5 CUKINGNAN, R. A.; CAREY, J. S.; WITTIG, J. H.; BROWN, B. G. - Influence of complete coronary revascularization on relief of angina. J. Thorac. Cardiovasc. Surg., 79: 188-193, 1980.

6 DILLEY, R. B.; CANNON, J. A.; KATTUS, A. A. - The treatment of coronary occlusive disease by endarterectomy. J. Thorac. Cardiovasc. Surg., 50: 511-526, 1965.

7 ELLIS, P. R. \& COOLEY, D. A. - The patch technique as an adjunct to coronary endarterectomy. J. Thorac. Cardiovasc. Surg., 42: 236-243, 1961.

8 GOLDSTEIN, J.; COOPER, E.; SALTUPS, A.; BOXALL, J. - Angiographic assessment of graft patency after coronary endarterectomy. J. Thoracic Cardiovasc. Surg., 102: 539-545, 1991.

9 GROVES, L. K.; LOOP, F. D.; SILVER, G. M. Endarterectomy as a supplement to coronary artery saphenous vein bypass surgery. J. Thorac. Cardiovasc Surg., 64: 514-522, 1972.

10 HALIM, M. A.; OUERESHI, S. A.; TOWERS, M. K.; YACOUB, M. H. - Early and late results of combined endarterectomy and coronary bypass grafting for diffuse coronary disease. Am. J. Cardiol., 49: 16231626, 1982.

11 HOCHBERG, M. S.; MERNILL, W. H.; MICHAELIS, L. L.; McINTOCH, C. L. - Results of combined coronary artery endarterectomy and coronary bypass for diffuse coronary artery disease. J. Thorac. Cardiovasc. Surg., 75: 38-46, 1978.
12 Jatene, F. B.; Lemos, P. C. P.; JATENE, A. D. Fluxômetro hemodinâmico em cirurgia de revascularização do miocárdio. Rev. Bras. Cir. Cardiovasc., 1: 49-53, 1986.

13 KEON, W.; AKYWREKLIY, O.; BEDARD, P. - Coronary endarterectomy: an adjunct to coronary artery bypass grafting. Surgery, 86: 859-867, 1979.

14 KEON, W.; HENCHY, P.; BOYD, W. D.; WALLEY, M V. - Long term follow-up of coronary endarterectomy. In: WALTER, P. J. (ed.) - Treatment of end-stage coronary artery disease. Advances in Cardiology. Basel: Karger, 1988, vol. 36, p. 106-126.

15 KLIE, J. H.; JOHNSON, L.; SMULYAN, H.; POTTS, J. L.; OBEID, A. L.; FHUEHAN, C. T.; EICH, R. H.; PARKER, F. B.; WEBB, W. R. - Gas endoarterectomy of right coronary artery: the importance of proximal bypass graft. Circulation, 49: 63-67, 1974.

16 LAWRIE, G. M.; MORRIS, G. C.; SILVERS, A. - The influence of residual disease after coronary bypass on the 5 year survival rate of 1274 men with coronary artery disease. Circulation, 66: 717-723, 1982.

17 LIVESAY, J. J. \& COOLEY, D. A. - The role and results of coronary endarterectomy. In: BAVE, A. E.,(ed.) Glenn's Thoracic and cardiovascular surgery. 5. ed. East Norwalk, Appleton \& Lange, 1991.p. 1791-1799.

LIVESAY, J. J.; COOLEY, D. A.; HALLMAN, G. L.; REUL, G. J.; OTT, D. A.; DUCAN, J. M.; FRAZIER, O. H. - Early and late results of coronary endarterectomy: analysis of 3.369 patients. J. Thorac. Cardiovasc. Surg., 92: 649-660, 1986.

19 LONGMIRE Jr., W.; CANNON, J. A.; KATTUS, A. A. Direct-vision coronary endarterectomy for angina pectoris. N. Engl. J. Med., 259: 993-999, 1958.

MILLER, D. C.; STINSON, E. B.; PHILIP, E. O.; REITZ, B. A.; JAMIESON, S. W.; MORENO-CABRAL, R. J.; SHUMWAY, N. E. - Long-term clinical assessment of the efficacy of adjunctive coronary endarterectomy. J. Thorac. Cardiovasc. Surg., 81: 21-29, 1981.

QUERESHI, S. A.; HALIM, M. A.; PILLAI, R.; SMITH, P.; YACOUB, M. H. - Endarterectomy of the left coronary system: analisis of a 10 years experience. J. Thorac. Cardiovasc. Surg., 89: 852-859, 1985.

SAWYER, P. N.; KAPLITT, M.; SOBEL, S.; KARLSON, K. E.; STUDKEY, J.; WECHSLER, B. M.; SUMMERS, O. N.; DENNIS, C. - Experimental and clinical experience with coronary gas endarterectomy. Arch. Surg., 95: 736-742, 1967.

23 SCHAFF, H. V.; GERSH, B. J.; PLUTH Jr., A. Survival and functional to 12 years after surgery in 500 patients. Circulation, 68 (Parte 2): 200204, 1983. 
SALERNO, P. R.; DINKHUYSEN, J. J.; CHACCUR, P.; ABDULMASSIH NETO, C.; SANTOS, M. A.; SOUZA, L. C. B.; JATENE, A. D. - Técnica e resultados da endarterectomia de artéria coronária: Rev. Bras. Cir. Cardiovasc., 9 (3): 152-158, 1994.

24 SONES Jr., F. M. \& SHIREY, E. K. - Cine coronary arteriography. Mod. Conc. Cardiovasc Dis., 31: 735, 1962.

25 VINEBERG, A. M. - Development of an anastomosis between the coronary vessels and a transplanted internal mammary artery. Canad. Med. Assoc. J., 55: 117, 1946.
VINEBERG, A. \& MILLER, G. - Internal mammary coronary anastomosis in the surgical treatment of coronary artery insuficiency. Canad. Med. Assoc. J., 64: 204, 1951.

27 WALLEY, U. M.; BYARD, R. W.; KEON, W. J. - A Study of the sequential morphologic change after manual coronary endarterectomy. Thoracic. Cardiovasc. Surg., 102: 890-894, 1991. 